\title{
New insights into mechanisms of Alzheimer's disease revealed by a dynamic functional magnetic resonance imaging study
}

\author{
Yicheng Long ${ }^{1,2,3 \wedge}$ \\ ${ }^{1}$ Department of Psychiatry, The Second Xiangya Hospital, Central South University, Changsha, China; ${ }^{2}$ Mental Health Institute of Central South \\ University, Changsha, China; ${ }^{3}$ China National Clinical Research Center on Mental Disorders, Changsha, China \\ Correspondence to: Yicheng Long, PhD, MD. Department of Psychiatry, The Second Xiangya Hospital, Central South University, 139 Middle Renmin \\ Road, Changsha 410011, China. Email: yichenglong@csu.edu.cn. \\ Response to: Li T, Liao Z, Mao Y, et al. Temporal dynamic changes of intrinsic brain activity in Alzheimer's disease and mild cognitive impairment \\ patients: a resting-state functional magnetic resonance imaging study. Ann Transl Med 2021;9:63.
}

Submitted Feb 15, 2021. Accepted for publication Apr 11, 2021.

doi: $10.21037 /$ atm-21-743

View this article at: http://dx.doi.org/10.21037/atm-21-743

We have read the study by Li et al. (1) with great interest and would like to congratulate the authors for the publication of this important study. Based on restingstate functional magnetic resonance imaging ( $\mathrm{fMRI}$ ), they compared the dynamic amplitude of low-frequency fluctuation (dALFF) and dynamic fraction amplitude of low-frequency fluctuation (dfALFF) among 111 patients with Alzheimer's disease (AD), 29 patients with mild cognitive impairment (MCI) and 73 healthy controls (HC). The findings suggest abnormal dynamic features of brain activity in $\mathrm{AD}$ patients, which are ignored by conventional static fMRI studies. It gives a new insight into the neurophysiological mechanisms of $\mathrm{AD}$. As such, there are a few points which we would like to bring up.

During the data preprocessing stage, global signal regression (GSR) was not performed in the current work. However, some previous studies have indicated that measures of the dynamic fluctuations in brain activity are sensitive to head motion (2), and GSR is one of the most effective denoising strategies to diminish motion artifacts (3). For such a reason, although being controversial considering that GSR could exacerbate the impacts of anti-correlations between brain regions (4), more adequate results may be obtained with GSR to minimize the motion-related effects in dynamic fMRI studies. In fact, in many of the recent research on dALFF or dfALFF, the primary analyses were performed with GSR (5-7). Therefore, the authors might benefit from adding complementary analysis with GSR to probe its possible effects on dALFF and dfALFF.

The AD patients showed significantly increased dALFF variabilities within regions of the cerebellum and temporal lobes when compared to HCs, while no significant differences were found between the MCI patients and HCs. Based on such results, the authors concluded that abnormally increased variabilities of brain activity within these regions can be recognized as dementia-specific processes. Nevertheless, it is noteworthy that in the current study, the sample size of MCI group $(n=29)$ is much smaller than those of the $\mathrm{AD}(\mathrm{n}=111)$ and $\mathrm{HC}(\mathrm{n}=73)$ groups. Since the reduction in sample size results in a lower statistical power for detecting true effects (8), it is possible that similar alterations are occurring in the MCI patients but can only be detected in a larger sample.

The current study was focused on dALFF and dfALFF, which are both voxel-based measures to assess the dynamic fluctuations of local brain activity. Beyond them, there are measures of brain dynamics with a larger scope, such as the temporal variability of functional connectivity $(8,9)$ and stability of modular structures (10) for large-scale brain networks. In my view, future studies are encouraged to investigate the associations between $\mathrm{AD}$ and functional brain dynamics by combining both the dALFF/dfALFF

^ ORCID: 0000-0003-4231-2136. 
and these network-level assessments. This is important since the aberrant dynamic features of brain function in neuropsychiatric disorders are often observed for the entire brain systems $(8,9)$.

\section{Acknowledgments}

Funding: This study was supported by the National Natural Science Foundation of China (grant number 82071506).

\section{Footnote}

Provenance and Peer Review: This article was a standard submission to the journal. The article did not undergo external peer review.

Conflicts of Interest: The author has completed the ICMJE uniform disclosure form (available at http://dx.doi. org/10.21037/atm-21-743). The author has no conflicts of interest to declare.

Ethical Statement: The author is accountable for all aspects of the work in ensuring that questions related to the accuracy or integrity of any part of the work are appropriately investigated and resolved.

Open Access Statement: This is an Open Access article distributed in accordance with the Creative Commons Attribution-NonCommercial-NoDerivs 4.0 International License (CC BY-NC-ND 4.0), which permits the noncommercial replication and distribution of the article with the strict proviso that no changes or edits are made and the original work is properly cited (including links to both the formal publication through the relevant DOI and the license). See: https://creativecommons.org/licenses/by-nc-nd/4.0/.

\section{References}

1. Li T, Liao Z, Mao Y, et al. Temporal dynamic changes

Cite this article as: Long Y. New insights into mechanisms of Alzheimer's disease revealed by a dynamic functional magnetic resonance imaging study. Ann Transl Med 2021;9(12):1031. doi: $10.21037 / \mathrm{atm}-21-743$ of intrinsic brain activity in Alzheimer's disease and mild cognitive impairment patients: a resting-state functional magnetic resonance imaging study. Ann Transl Med 2021;9:63.

2. Laumann TO, Snyder AZ, Mitra A, et al. On the Stability of BOLD fMRI Correlations. Cereb Cortex 2017;27:4719-32.

3. Lydon-Staley DM, Ciric R, Satterthwaite TD, et al. Evaluation of confound regression strategies for the mitigation of micromovement artifact in studies of dynamic resting-state functional connectivity and multilayer network modularity. Netw Neurosci 2019;3:427-54.

4. Murphy K, Fox MD. Towards a consensus regarding global signal regression for resting state functional connectivity MRI. Neuroimage 2017;154:169-73.

5. Liao W, Li J, Ji GJ, et al. Endless Fluctuations: Temporal Dynamics of the Amplitude of Low Frequency Fluctuations. IEEE Trans Med Imaging 2019;38:2523-32.

6. Liang $Y$, Jiang $X$, Zhu W, et al. Disturbances of Dynamic Function in Patients With Bipolar Disorder I and Its Relationship With Executive-Function Deficit. Front Psychiatry 2020;11:537981.

7. Cui Q, Sheng W, Chen Y, et al. Dynamic changes of amplitude of low-frequency fluctuations in patients with generalized anxiety disorder. Hum Brain Mapp 2020;41:1667-76.

8. Long Y, Cao H, Yan C, et al. Altered resting-state dynamic functional brain networks in major depressive disorder: Findings from the REST-meta-MDD consortium. Neuroimage Clin 2020;26:102163.

9. Long Y, Liu Z, Chan CKY, et al. Altered Temporal Variability of Local and Large-Scale Resting-State Brain Functional Connectivity Patterns in Schizophrenia and Bipolar Disorder. Front Psychiatry 2020;11:422.

10. Long Y, Chen C, Deng M, et al. Psychological resilience negatively correlates with resting-state brain network flexibility in young healthy adults: a dynamic functional magnetic resonance imaging study. Ann Transl Med 2019;7:809. 